feeling of life-weariness as risk factors of sleep duration, and to evaluate how the relationship between sleep duration and these psychosocial factors influences the risk of death.

Methods Baseline study using a structured questionnaire was performed in $1986 / 1987$ on a random sample $(n=2605)$ of older people aged 65 years and over, community-dwelling citizens of Krakow. Vital status was monitored over 22 years. The analysis was performed using logistic regression and Cox hazard models.

Results The mean observation period of the cohort was 11.5 years, for a total of 29951 person-years. 1341 deaths in women and 771 in men were recorded. Strong feeling of life-weariness was found as predictor of short sleep in both genders. Poor self-rated health in women and both low functional activity and low level of education in men was associated with a long sleep. Analysis showed increased risk of death in men who slept $<7 \mathrm{~h}$ and reported a strong feeling of life-weariness ( $\mathrm{HR}=2.2,95 \% \mathrm{CI} 1.2$ to 4.1), for women higher mortality was observed in those who reported long sleep and poor self-rated health ( $\mathrm{HR}=1.6,95 \%$ CI 1.2 to 2.2 ).

Conclusions Results suggest that sleeping patterns in elders depending on several psychosocial conditions influence the mortality patterns in older age.

\section{P1-550 SELF-RATED HEALTH AND MORTALITY IN A PROSPECTIVE CHINESE ELDERLY COHORT}

doi:10.1136/jech.2011.142976h.37

1J Zhou, ${ }^{1} \mathrm{~J}$ Johnston, ${ }^{2} \mathrm{~W}$ M Chan, ${ }^{2} \mathrm{Y}$ F Hui, ${ }^{2} \mathrm{P}$ S Ng, ${ }^{1} \mathrm{~T}$ H Lam, ${ }^{1} \mathrm{M}$ Schooling. ${ }^{*}$ ${ }^{1}$ Department of Community Medicine and School of Public Health, Li Ka Shing Faculty of Medicine, The University of Hong Kong, Hong Kong; ${ }^{2}$ Department of Health, The
Government of the Hong Kong Special Administrative Region, Hong Kong SAR, People's Republic of China, Hong Kong

Background Most previous studies of self-rated health (SRH) and mortality are in western populations and use global SRH. Conceptualisations of health and the cause-composition of mortality may be context specific; we examined the association of SRH with allcause and cause-specific mortality in a developed non-western population.

Methods We used multivariable Cox regression to examine, in 64189 Chinese people aged $65+$ years enrolled at 18 Elderly Health Centres from 1998 to 2001, the adjusted associations of age-comparative and self-comparative SRH with mortality and whether the associations varied by follow-up duration, sex, age or socio-economic position.

Results After a mean follow-up of 8.2 years, there were 12350 deaths. Worse age-comparative SRH was associated with higher mortality (HR 1.73, 95\% CI 1.62 to 1.85 ) than better SRH, adjusted for sex, age, education, housing, monthly expenditure, smoking, alcohol use, body mass index and physical activity. The associations varied by sex in the short term and weakened with age. Agecomparative SRH was more strongly associated with mortality from circulatory diseases (HR 1.93, 95\% CI 1.70 to 2.18) and respiratory diseases (HR 2.08, 95\% 1.79 to 2.42) than from cancer (HR 1.27, $95 \%$ CI 1.13 to 1.43 ). Worse self-comparative SRH was not associated with higher mortality.

Conclusion Age-comparative SRH is a good predictor of mortality in older people from a non-western setting but self-comparative $\mathrm{SRH}$ is not. However, the association was less marked than in western settings possibly because of contextually specific assessments of health or a different pattern of mortality. 